\title{
The effect of a fibre extract from the red seaweed, Palmaria palmata, on lipid metabolism and inflammation in healthy adults
}

\author{
Zoe Irwin ${ }^{1}$, Emeir M. McSorley ${ }^{2}$, Mary M. Slevin $^{2}$, Lisa Rowan $^{2}$, Paul McMillen $^{2}$, \\ Danielle McCullagh ${ }^{2}$, Pamela J. Magee ${ }^{2}$, Christopher I. R. Gill ${ }^{2}$, Paul Cherry ${ }^{2}$, William Crowe ${ }^{2}$, \\ Conall R. Strain ${ }^{3}$, Catherine Stanton ${ }^{3}$ and Philip J. Allsopp ${ }^{2}$ \\ ${ }^{1}$ Nutrition Innovation Centre for Food and Health, Ulster University, Portstewart, United Kingdom, \\ ${ }^{2}$ Nutrition Innovation Centre for Food and Health, Ulster University, Coleraine, United Kingdom and \\ ${ }^{3}$ Teagasc Food Research Centre, Moorepark, Cork, Ireland
}

\begin{abstract}
Evidence from observational studies indicates that seaweed consumption may reduce the risk of non-communicable diseases such as cardiovascular disease, type two diabetes, and obesity. Accumulating evidence from in vitro and animal studies suggest seaweed have antihyperlipidemic, anti-inflammatory and antioxidant properties which may in part be attributed to the high content of soluble dietary fibre in seaweeds. The viscosity of seaweed fibres is suggested to mediate antihyperlipdiemic effects via the alteration of lipid/bile acid absorption kinetics to decrease low-density lipoprotein cholesterol (LDL). Thus, there is a need to evaluate the efficacy of seaweed derived dietary fibre in the management of dyslipidemia. Therefore, the aim of this study was to determine the effect of a fibre rich extract from Palmaria palmata on the lipid profile as well as markers of inflammation and oxidative stress in healthy adults. A total of 60 healthy participants ( 30 male and 30 female) aged 20 to 58 years, were assigned to consume the Palmaria palmata fibre extract (5g/day), Synergy-1 and the placebo (maltodextrin) for a duration of 4 weeks with a minimum 4 week washout between each treatment in a double blind, randomised crossover study conducted over 5 months. Fasting concentrations of cholesterol, triglycerides and high-density lipoprotein cholesterol (HDL) were analysed and low-density lipoprotein cholesterol (LDL) and LDL: HDL ratio was calculated. C-reactive protein (CRP) and Ferric Reducing Ability of Plasma (FRAP) were analysed as markers of inflammation and oxidative stress, respectively. Supplementation for 4 weeks with Palmaria palmata resulted in favourable changes to lipid profiles with a reduced LDL:HDL ratio; however intention-to-treat univariate ANCOVA identified no significant difference between the treatment groups over time on any of the lipid profile markers. A non-significant increase in CRP and triglyceride concentration along with lower FRAP was also observed with Palmaria palmata supplementation. Evidence from this study suggests that Palmaria palmata may have effects on lipid metabolism and appears to mobilise triglycerides. More research is needed in individuals with dyslipidaemia to fully elucidate these effects.
\end{abstract}

\section{Conflict of Interest}

There is no conflict of interst. 